\title{
ANÁLISIS DE LIGAMIENTO DE ESQUIZOFRENIA CON GENES DEL NEURODESARROLLO MEDIANTE CINCO MARCADORES MICROSATÉLITES
}

\author{
LINKAGE ANALYSIS OF SCHIZOPHRENIA AND NEURODEVELOPMENTAL \\ GENES USING FIVE MICROSATELLITE MARKERS
}

\author{
A. MIRANDA, G. BEDOYA, O. CAMPO, J. GARCÍA, C. PALACIO, C. LÓPEZ, \\ J. CALLE, M. LÓPEZ, A. RUIZ y J. OSPINA-DUQUE*
}

\section{RESUMEN}

Introducción. El objetivo fue evaluar el efecto que posibles variantes en genes del neurodesarrollo tienen sobre la susceptibilidad a la esquizofrenia en dos familias colombianas.

Métodos. En dos familias con varios afectados por esquizofrenia según DSM-IV, se depuró el fenotipo con la entrevista diagnóstica para estudios genéticos (DIGS) y el procedimiento de "mejor estimación diagnóstica". Se evaluó el poder de ligamiento en las familias mediante análisis de simulación con el programa SLINK y se hizo análisis de ligamiento paramétrico y no-paramétrico con marcadores microsatélites D7S2504 situado en el gen RELN, D6S2414 y D6S1014 cercanos al gen NOTCH4 y D1S1653 y D1S1679.

Resultados. Con la simulación se obtuvo un lod score máximo de 1.33 para las dos familias cuando se consideró el fenotipo de forma estrecha con patrón de herencia autosómico recesivo. El lod score máximo obtenido con el análisis de ligamiento fue 0.18 para el marcador D7S2504 (situado en el gen de la relina) con patrón de herencia autosómico recesivo, con penetrancia incompleta y al considerar el fenotipo amplio. Para este mismo marcador y con fenotipo amplio, se obtuvo el valor más bajo de $p(p=0.4)$ en el análisis no paramétrico, correspondiente a un NPL de pares de 0.03 .

Conclusión. En estas familias no fue posible confirmar ni descartar ligamiento entre la esquizofrenia y los marcadores D1S1653, D1S1679, D6S2414, D6S1014 y D7S2504 con el análisis paramétrico y no paramétrico, ya que no se encontraron lod score menores de -2.

Palabras-Clave: Esquizofrenia, genética, ligamiento, neurodesarrollo. 
ABSTRACT

Introduction. The goal was to evaluate the effect that possible variants in neurodevelopmental genes have on the susceptibility to schizophrenia in two Colombian families.

Methodology. In two families with more than two affected members with schizophrenia using DSM-IV criteria, the phenotype was defined with the Diagnostic Interview for Genetic Studies (DIGS), and the Best Estimate procedure. The linkage power was evaluated in the families through simulation analysis with the SLINK software and a parametric and nonparametric linkage analysis was made with the micro-satelite markers D7S2504 situated at the RELN gene, the D6S2414 and D6S1014 near the NOTCH4 gene, and D1S1653 and D1S1679.

Results. A maximum lod score of 1.33 was obtained for both families with the simulation when the phenotype was considered in a very strict form and using an autosomic recesive inheritance patron. The maximum obtained lod score with the linkage analysis was 0.18 for the D7S2504 marker (located at the reelin gene) with a recesive autosomic inheritance patron, an incomplete penetrance and considering a broader phenotype. For this marker and with a broader phenotype, a lower $p$ value $(p=0.4)$ was obtained in the non-parametric analysis, corresponding to a NPL of 0.03 .

Conclusion. In these families it was not possible to discard linkage between schi-zophrenia and the D1S1653, D1S1679, D6S2414, D6S1014 and D7S2504 markers with the parametric and non parametric analysis, because no lod score values lower than -2 were found.

KEY words: Schizophrenia, genetics, linkage, neurodevelopment.

\section{INTRODUCCIÓN}

La esquizofrenia es un síndrome psiquiátrico heterogéneo, crónico, seriamente incapacitante y con altos costos personales, sociales y económicos. ${ }^{1,2}$ Existen evidencias que respaldan el componente genético como un factor importante en su etiología, tales como la presencia de varios afectados en grupos familiares de poblaciones diferentes, los estudios de adopción y de concordancia gemelar. Con análisis de segregación compleja se ha inferido que la esquizofrenia tiene un patrón de herencia multifactorial. ${ }^{3}$ Según este patrón de herencia un conjunto de variantes génicas interactúan entre sí y con el ambiente y generan susceptibilidad al trastorno. ${ }^{4}$ Para investigar cuáles podrían ser esas va- riantes génicas, se han estudiado genes que codifican proteínas que participan en la neurotransmisión. ${ }^{5,6}$ En los últimos años, se han realizado varios trabajos que han partido de la hipótesis del neurodesarrollo, la cual sugiere que la herencia de polimorfismos en genes involucrados en la formación del cerebro en las etapas pre y postnatal origina una estructura cerebral anormal que conlleva a una susceptibilidad para el síndrome. Esta susceptibilidad se expresa mediante alteraciones bioquímicas, neurofisiológicas, neuroanatómicas y comportamentales que pueden evolucionar o no a esquizofrenia. ${ }^{7,8}$ Esta hipótesis está respaldada por estudios neuropatológicos y neuroimaginológicos, la presencia de alteraciones premórbidas comportamentales, intelectuales y neurológicas y 
la consegregación con esquizofrenia de anomalías físicas menores. ${ }^{9}$ A este respecto, en los genes RELN y NOTCH4, que participan en el desarrollo cerebral, se ha encontrado variantes génicas asociadas a esquizofrenia. $^{10-12}$

El gen RELN está ubicado en la región $7 q 22$ y codifica una glicoproteína llamada relina que participa en el posicionamiento $\mathrm{y}$ migración de neuronas durante el neurodesarrollo y en la transmisión sináptica junto con otras señales. ${ }^{13,14}$ Esto fue dilucidado gracias a los estudios en un modelo murino de relina, el ratón "enrollador" (releer) que se caracteriza por incoordinación motora, temblor y ataxia por defectos en el posicionamiento normal de las neuronas en la corteza cerebral y cerebelar. Con este modelo se logró determinar que la deficiencia en la función de la relina puede suspender la migración neuronal y promover la laminación cortical anormal. ${ }^{15}$ En los humanos la relina se ha visto implicada en la etiología de trastornos como esquizofrenia, trastorno afectivo bipolar, autismo y lisencefalia. ${ }^{16}$ Basándose en la observación de una menor expresión de relina en las cortezas temporales de individuos esquizofrénicos, se han enfocado varias investigaciones en la regulación de la expresión del gen. Chen y cols. en el 2002 detectaron una transversión Go $\mathrm{C}$ en la posición -888 , pero ni las frecuencias alélicas y genotípicas no presentaron diferencias significativas entre casos y controles. ${ }^{17,}{ }^{18} \mathrm{Sin}$ embargo este resultado no descarta la presencia de mutaciones en regiones involucradas en la regulación de la expresión del gen como promotores, regiones 5' y 3' no traducidas y sitios críticos para el procesamiento del RNA mensajero (splicing).

El gen NOTCH4 participa en el neurodesarrollo de la Drosophila Melanogaster y en la neurogénesis y gliogénesis de murinos.
Basados en esta evidencia y en su localización en la región 6p21.3, en la cual se han obtenido gran número de hallazgos de ligamiento significativo a esquizofrenia, se han realizado estudios de asociación del gen NOTCH4 con el trastorno, los cuales han dado resultados contradictorios. ${ }^{19,} 20$ Se ha reportado un fuerte desequilibrio de ligamiento entre un polimorfismo de repeticiones (CTG)n en el exon 1 del gen y la esquizofrenia. ${ }^{20}$ También se ha informado asociación entre variantes alélicas de este gen (repeticiones de CTG) y alteraciones en el volumen y la función del lóbulo frontal. ${ }^{12}$

En general, los estudios de ligamiento que se han realizado hasta el momento, no han podido producir resultados inequívocos $\mathrm{y}$ replicables. Sin embargo, hay evidencias importantes para las siguientes regiones: 22q11-12, 6p24-22, 6q, 8p22-21, 13q14, 1-q32 y 1q21-q22. ${ }^{21}$ Esta última región es de especial importancia, pues en ella se ha reportado uno de los lod score más altos para esquizofrenia (multipuntual $=6.5, \mathrm{p}=0.0002$; bipuntual=5.79, p<0.0002) entre los marcadores D1S1653 y D1S1679. ${ }^{22}$ En otros estudios se evaluaron 80 familias nucleares de origen español y encontraron una distorsión en la transmisión de alelos del D1S1679 en los sujetos con esquizofrenia, utilizando como fenotipo el síndrome en general y las dimensiones sintomáticas. Bajo la segunda aproximación se encontró asociación principalmente con el síndrome de distorsión de la realidad caracterizado por alucinaciones y delirios. ${ }^{23}$ Actualmente, existen al menos tres genes localizados en esta área que podrían con-siderarse como genes candidatos para esqui-zofrenia: Un canal neuronal de potasio activado por calcio de conductancia menor (KNNC3/hSKCa3); un gen que codifica para un recep-tor de alta afinidad para el factor de crecimiento nervioso (TRKA/NTRK1) y un regulador del gen de señalización de proteína 
G. ${ }^{23}$ Los canales de potasio activados por calcio juegan un papel muy importante en el patrón de disparo de las neuronas, por tanto las alteraciones en el canal hSKCa3 podrían afectar la excitabilidad neuronal e involucrarse en el espectro de síntomas de distorsión de la realidad. Por otro lado, la alteración de factores neutrópicos y sus receptores podrían alterar la formación y podado de sinapsis en el proceso de desarrollo prenatal y postnatal. ${ }^{24-26}$

Con la evidencia de un componente genético importante en la esquizofrenia y basados en la hipótesis del neurodesarrollo, decidimos evaluar el efecto que posibles variantes en genes del neurodesarrollo tienen sobre la susceptibilidad a la esquizofrenia en dos familias colombianas, mediante análisis de ligamiento con marcadores microsatélites D1S1653 y D1S1679 porque han presentado un lod score alto, D7S2504 situado en el gen RELN, y D6S2414 y D6S1014 cercanos al gen NOTCH4.

\section{MÉTODOS}

En los registros del Hospital Mental de Antioquia, el Hospital San Vicente de Paúl y la Clínica Sameín de la ciudad de Medellín, Colombia, se identificaron sujetos con diagnóstico de esquizofrenia. Una trabajadora social contactó al paciente y al menos algún familiar cercano para explicarles los objetivos del estudio, y los citó para realizar los siguientes procedimientos, previa firma del consentimiento informado:

1. Verificación de origen del paciente mediante una encuesta histórica.

2. Entrevista diagnóstica para estudios genéticos (DIGS 3.0) 27:28 $^{2}$ con el fin de confirmar o descartar esquizofrenia $u$ otros trastornos psiquiátricos en el paciente y los familiares. Como parte de la realización del DIGS, el psiquiatra revisó la historia clínica e interrogó a los familiares del sujeto que pudieran dar información sobre su patología. El diagnóstico fue reconfirmado mediante un procedimiento de Mejor Estimación Diagnóstica (Best Estimate), en el cual los registros del DIGS se reevaluaron por tres psiquiatras expertos en trastornos psicóticos. Cada uno de ellos hizo el diagnóstico por separado, cuando todos estuvieron de acuerdo se consideró confirmado. Cuando no fue así, los tres expertos se reunieron y obtuvieron el diagnóstico por consenso. El diagnóstico se hizo según criterios del DSM-IV. ${ }^{1}$

3. Se tomó una muestra de $20 \mathrm{cc}$ de sangre periférica a los pacientes y sus familiares, de ésta se extrajo DNA por el método de fenol-cloroformo. A partir del DNA se amplificaron por PCR los marcadores D1S1679, D1S1653, D6S1014, D6S2414 y D7S2504 utilizando uno de los cebadores marcado con fluorocromos. La secuencia de los cebadores utilizados está disponible en las bases de datos públicas. Para la estandarización de la amplificación se utilizaron los protocolos disponibles en las bases de datos públicas GENBANK y GDB. La determinación de los tamaños alélicos se realizó por electroforesis capilar en un analizador genético ABIPRISMA 310 y mediante los programas GENSCAN y GENOTYPER.

\section{ANÁLISIS DE LOS DATOS}

Simulación. En las familias extendidas se evaluó el poder mediante simulación con el programa SLINK del paquete LINKAGE, versión 4.91 bajo los patrones de herencia autosómica dominante y recesivo y con dos modelos diagnósticos que resultan en dos fenotipos amplio y estrecho. En el fenotipo 
amplio se consideraron como afectados todos los individuos con patología psiquiátrica. En el estrecho se consideraron como afectados todos los individuos con patología psiquiátrica. En el estrecho se tomaron como afectados únicamente quienes tenían diagnóstico de esquizofrenia o trastorno esquizoafectivo. Las penetrancias utilizadas fueron aquellas calculadas por Brzustowicz y col., las cuales varían de acuerdo con el patrón de herencia y el fenotipo utilizado. ${ }^{22}$ Las frecuencias alélicas fueron inferidas para cada fenotipo y patrón de herencia, a partir de la prevalencia de enfermedad del 1\%, mediante las siguientes fórmulas:

Patrón de herencia autosómico dominante: $\mathrm{q}=\mathrm{I}(2 / \mathrm{f})$

Patrón de herencia autosómico recesivo: $\mathrm{q}=\ddot{\mathrm{O}}(\mathrm{I} / \mathrm{f})$

Donde, $\mathrm{q}=$ frecuencia del alelo mutado, $\mathrm{I}=$ prevalencia, $\mathrm{f}=$ penetrancia.

Por lo tanto, los datos de frecuencia alélica de la enfermedad y las penetrancias utilizadas fueron las siguientes:

Dominante amplio: $\mathrm{q}=0.007$, fAA $=0.9$, $\mathrm{fAa}=0.8$, faa $=0.009$

Dominante estrecho: $\mathrm{q}=0.005$, fAA $=0.75$, $\mathrm{fAa}=0.5$, faa $=0.001$

Recesivo amplio: $\mathrm{q}=0.12, \mathrm{fAA}=0.01$, fAa $=0.01$, faa $=0.6$

Recesivo estrecho: $\mathrm{q}=0.1$, fAA $=0.0015$, $\mathrm{fAa}=0.0015$, faa $=0.6$

Se asumió un marcador con cuatro alelos de igual frecuencia cada uno (25\%), una tasa de fenocopias del $10 \%$ y una heterogeneidad genética del $20 \%$.

Análisis de ligamiento paramétrico y no paramétrico. Con los genotipos obtenidos se realizó análisis de ligamiento paramétrico bipuntual entre cada uno de los marcadores y el locus putativo de la en- fermedad utilizando el programa LINKAGE versión 5.2. Las frecuencias alélicas de los cinco marcadores en la población, se obtuvieron por genotipificación de 49 individuos sanos de origen antioqueño recolectados previamente utilizando el programa GENPOP versión 3.3. El análisis se hizo bajo patrones de herencia autosómico dominante y recesivo con fenotipos amplio y estrecho, se asumieron los mismos valores de penetrancia y frecuencia del alelo mutado que se obtuvieron en los resultados de la simulación.

Para el fenotipo amplio se realizó además, análisis de ligamiento no paramétrico por el método de pares de familiares afectados (ARP) en el cual, basados en el estadístico NPL, se comparan los alelos idénticos por descendencia (IBD), compartidos por los familiares afectados, para determinar si hay diferencia entre las frecuencias esperadas y observadas. El estadístico NPL se utilizó de dos formas, comparando alelos entre los diferentes pares de individuos afectados (NPL pairs) y comparando todos los individuos afectados simultáneamente (NPL all). Este análisis fue realizado con el programa MERLÍN versión 0.0.8 (Multipoint Engine for Rapid Likelihood Inference).

\section{RESULTADOS}

\section{Caracterización de las familias}

Se recolectaron dos familias con agregación familiar de la enfermedad, ambas originarias del mismo municipio de Antioquia a las cuales se denominaron familia 1 y familia 2 . Las dos familias en conjunto poseen 31 individuos. En la familia 1 se diagnosticaron cinco miembros para fenotipo estrecho (Dos con trastorno esquizoafectivo y tres con esquizofrenia) y para el amplio se diagnosticaron ocho. En la familia 2 se encontró la esquizofrenia como único trastorno psiquiátrico en tres individuos, uno de ellos fallecido. 


\section{Simulación}

El máximo lod score que se obtuvo por el análisis de simulación fue de 1.33 cuando se utilizaron las dos familias (Tabla 1). Así mismo, este análisis predice que la probabilidad de obtener un lod score de 1 es del $5 \%$ y de 2 ó 3 es de 0 (Tabla 2). Dado que en las simulaciones no se encontraron lod score promedios menores de -2 , que es el parámetro utilizado para rechazar ligamiento en enfermedades complejas se decidió que era necesario extender más las familias para aumentar su poder de detectar ligamiento o rechazarlo. Sin embargo no se pudo realizar la evaluación de otros posibles afectados porque no aceptaron participar en la investigación.

TABLA 1

MÁXIMO LOD SCORE OBTENIDO EN EL ANÁLISIS DE SIMULACIÓN EN DOS FAMILIAS ANTIOQUEÑAS

\begin{tabular}{|l|c|c|c|c|}
\hline $\begin{array}{l}\text { Patrón de } \\
\text { herencia y modo } \\
\text { diagnóstico }\end{array}$ & FAMILIA & $\begin{array}{c}\text { Máximo lod score } \\
\text { promedio }\end{array}$ & $\begin{array}{c}\text { Mínimo lod score } \\
\text { obtenido }\end{array}$ & $\begin{array}{c}\text { Máximo lod score } \\
\text { obtenido }\end{array}$ \\
\hline $\begin{array}{l}\text { Autosómico } \\
\text { dominante, } \\
\text { fenotipo amplio }\end{array}$ & FAMILIA 1 & 0.17 & 0 & 0.93 \\
\cline { 2 - 5 } & FAMILIA 2 & 0 & 0 & 0 \\
\hline $\begin{array}{l}\text { Autosómico } \\
\text { dominante, } \\
\text { fenotipo estrecho }\end{array}$ & FAMBLIA 1 & 0.17 & 0 & 0.93 \\
\cline { 2 - 5 } & FAMILIA 2 & 0.067 & 0 & 0.72 \\
\hline $\begin{array}{l}\text { Autosómico, } \\
\text { recesivo, } \\
\text { fenotipo amplio }\end{array}$ & FAMILIA 1 & 0.067 & 0 & 0.72 \\
\cline { 2 - 5 } & FAMILIA 2 & 0.056 & 0 & 0.67 \\
\hline $\begin{array}{l}\text { Autosómico } \\
\text { recesivo, } \\
\text { fenotipo estrecho }\end{array}$ & FAMBAS & 0.12 & 0 & 0.79 \\
\cline { 2 - 5 } & FAMILIA 2 & 0.35 & 0 & 1.22 \\
\cline { 2 - 5 } & AMBAS & 0.06 & 0 & 0.13 \\
\hline
\end{tabular}

TABLA 2

PRobabilidad DE OBTENER UN LOD SCORE DE 1, 2 Ó 3 EN DOS FAMILIAS ANTIOQUEÑAS

\begin{tabular}{|c|c|c|c|c|}
\hline LOD SOCRE & \multicolumn{4}{|c|}{ PROBABILIDAD (\%)* } \\
\hline & $\begin{array}{c}\text { Dominante } \\
\text { amplio }\end{array}$ & $\begin{array}{c}\text { Recesivo } \\
\text { amplio }\end{array}$ & $\begin{array}{c}\text { Dominante } \\
\text { estrecho }\end{array}$ & $\begin{array}{c}\text { Recesivo } \\
\text { estrecho }\end{array}$ \\
\hline 1 & 0 & 0 & 0 & 5 \\
\hline 2 & 0 & 0 & 0 & 0 \\
\hline 3 & 0 & 0 & 0 & 0 \\
\hline
\end{tabular}

* Poder calculado para ambas familias 
Como el análisis de simulación es una aproximación hipotética basada en la asignación aleatoria de genotipos a los individuos de las familias evaluadas, los resultados no siempre son el fiel reflejo de la información que se ha de obtener con el análisis de ligamiento y dado que visualmente se observaba que al menos en la familia 1 , en uno de sus núcleos familiares estaban los padres disponibles y mostraba varios individuos afectados que podían arrojar información de ligamiento se decidió proseguir con las evaluaciones y genotipificaciones.

\section{Análisis de ligamiento paramétrico y no paramétrico}

Mediante el análisis de ligamiento bipuntual se obtuvieron los valores máximos de lod score de 0.18 con $\theta=0.1$ y 0.13 con $\theta=0.2$, para el marcador D7S2504 bajo el patrón de herencia autosómico recesivo, con penetrancia incompleta y fenotipo amplio. El menor lod score se obtuvo para el marcador D1S1679 ( -0.74 con $\theta=0.1)$ (Tablas 3 y 4). Estos resultados concuerdan con el análisis no paramétrico, puesto que a pesar de que las diferencias entre lo esperado y lo observado con respecto a los alelos compartidos idénticos por descendencia (IBD) no fueron significativas, se obtuvo la probabilidad menor de la prueba de (0.4) para el mismo marcador que dio lod score máximo de 0.18 (D7S2504), con un NPLpairs de 0.03 (Tabla 5).

\section{DISCUSIÓN}

Se evaluaron cinco marcadores microsatélites en dos familias originarias del mismo municipio de Antioquia (Colombia) con agregación familiar de esquizofrenia. El máximo lod score obtenido fue de 0.18 para el locus D7S2504 a un $\theta=0.1$ bajo la definición amplia del fenotipo y con un patrón de herencia autosómico recesivo. Teniendo en

TABLA 3

SCORE BIPUNTUAL BAJO PATRÓN DE HERENCIA AUTOSÓMICO DOMINANTE CON PENETRANCIA INCOMPLETA PARA LOS MARCADORES D1S1679, D1S1653, D6S1014, D6S2414 y D7S2504 EN DOS FAMILIAS ANTIOQUEÑAS

\begin{tabular}{|l|l|c|c|c|c|c|}
\hline \multirow{2}{*}{ MARCADOR } & \multicolumn{7}{|c|}{$\mathbf{Z}(\boldsymbol{\theta})$} \\
\cline { 2 - 7 } & Modelo diagnóstico & $\boldsymbol{\theta = 0 . 1}$ & $\boldsymbol{\theta = 0 . 2}$ & $\boldsymbol{\theta}=\mathbf{0 . 3}$ & $\boldsymbol{\theta = 0 . 4}$ & $\boldsymbol{\theta}=\mathbf{0 . 5}$ \\
\hline \multirow{3}{*}{ D1S1679 } & Modo amplio & 0 & 0 & 0 & 0 & 0 \\
\cline { 2 - 7 } & Modo estrecho & -0.01 & -0.01 & 0 & 0 & 0 \\
\hline \multirow{2}{*}{ D6S1014 } & Modo amplio & -0.01 & -0.01 & 0 & 0 & 0 \\
\cline { 2 - 7 } & Modo estrecho & 0 & 0 & 0 & 0 & 0 \\
\hline \multirow{2}{*}{ D6S2414 } & Modo amplio & 0.01 & 0 & 0 & 0 & 0 \\
\cline { 2 - 7 } & Modo estrecho & 0 & 0 & 0 & 0 & 0 \\
\hline \multirow{2}{*}{ D7S2504 } & Modo amplio & -0.02 & -0.01 & 0 & 0 & 0 \\
\cline { 2 - 7 } & Modo estrecho & 0 & 0 & 0 & 0 & 0 \\
\cline { 2 - 7 } & Modo amplio & -0.09 & -0.05 & -0.03 & -0.01 & 0 \\
\hline
\end{tabular}


TABLA 4

SCORE BIPUNTUAL BAJO PATRÓN DE HERENCIA AUTOSÓMICO RECESIVO CON PENETRANCIA INCOMPLETA PARA LOS MARCADORES D1S1679, D1S1653, D6S1014, D6S2414 y D7S2504 EN DOS FAMILIAS ANTIOQUEÑAS

\begin{tabular}{|l|l|c|c|c|c|c|}
\hline \multirow{2}{*}{ MARCADOR } & \multicolumn{7}{|c|}{$\mathbf{Z}(\boldsymbol{\theta})$} \\
\cline { 2 - 7 } & Modelo diagnóstico & $\boldsymbol{\theta = \mathbf { 0 . 1 }}$ & $\boldsymbol{\theta = \mathbf { 0 . 2 }}$ & $\boldsymbol{\theta = \mathbf { 0 . 3 }}$ & $\boldsymbol{\theta}=\mathbf{0 . 4}$ & $\boldsymbol{\theta}=\mathbf{0 . 5}$ \\
\hline \multirow{3}{*}{ D1S1679 } & Modo amplio & -0.01 & -0.01 & 0 & 0.01 & 0 \\
\cline { 2 - 7 } & Modo estrecho & -0.74 & -0.35 & -0.14 & -0.03 & 0 \\
\hline \multirow{2}{*}{ D6S1014 } & Modo amplio & -0.17 & -0.07 & -0.02 & 0 & 0 \\
\cline { 2 - 7 } & Modo estrecho & -0.25 & -0.1 & -0.04 & -0.01 & 0 \\
\hline \multirow{2}{*}{ D6S2414 } & Modo amplio & 0.02 & 0.01 & 0.01 & 0 & 0 \\
\cline { 2 - 7 } & Modo estrecho & -0.16 & -0.03 & 0 & 0 & 0 \\
\hline & Modo amplio & 0.08 & 0.04 & 0.02 & 0.01 & 0 \\
\cline { 2 - 7 } & Modo estrecho & 0.04 & 0.03 & 0.02 & 0.01 & 0 \\
\cline { 2 - 7 } & Modo amplio & $\mathbf{0 . 1 8}$ & 0.13 & 0.07 & 0.02 & 0 \\
\hline
\end{tabular}

TABLA 5

ANÁLISIS DE LIGAMIENTO NO PARAMÉTRICO BIPUNTUAL (NPL PAIRS Y NPL ALL) BAJO MODO DIAGNÓSTICO AMPLIO

\begin{tabular}{|l|r|r|r|c|}
\hline \multirow{2}{*}{ Marcador } & \multicolumn{2}{|c|}{ NPL pairs } & \multicolumn{2}{c|}{ MPL all } \\
\cline { 2 - 5 } & LOD & P & \multicolumn{1}{c|}{ LOD } & P \\
\hline D1S1653 & -0.05 & 0.7 & -0.02 & 0.6 \\
\hline D1S1679 & -0.05 & 0.7 & -0.02 & 0.6 \\
\hline D6S1014 & -0.06 & 0.7 & -0.03 & 0.6 \\
\hline D6S2414 & -0.06 & 0.7 & -0.03 & 0.6 \\
\hline D7S2504 & 0.03 & 0.4 & 0.03 & 0.6 \\
\hline
\end{tabular}

cuenta que el menor lod score para los demás loci fue de -0.74 , no se puede descartar la existencia de ligamiento a estos loci ya que para enfermedades complejas el valor de lod score negativo para ligamiento es menor de -2 . El valor de lod score de 0.13 es sugestivo de ligamiento a la región del marcador D7S2504, lo cual concuerda con los resultados del análisis no paramétrico, pues a pesar de que no se obtuvo ligamiento, este marcador mostró la menor probabilidad en el análisis. Sin embargo, estos resultados deben tomarse con cautela dado que el análisis de ligamiento no fue concluyente y el NPL, que es más útil cuando se tiene información de múltiples marcadores en una región dada y es más exigente para evitar falsos positivos, tiene menos poder para detectar verdadero 
ligamiento en comparación con las estrategias paramétricas.

Los factores que afectan el poder para detectar ligamiento y/o asociación en las enfermedades complejas, pueden relacionarse con la población estudiada y con las características de los marcadores utilizados. Entre los primeros, se encuentra el tamaño de la muestra y la presencia de heterogeneidad genética. Entre los segundos están: la frecuencia de recombinación entre el locus de la enfermedad y el locus marcador, las frecuencias alélicas del marcador y su capacidad informativa y la especificación diagnóstica del fenotipo a estudiar. ${ }^{29}$ Todos estos factores pudieron influir, a favor o en contra, para detectar ligamiento en las familias evaluadas. Aunque en esta investigación desde el análisis de simulación se sospechaba que las familias no eran suficientemente informativas para detectar ligamiento, se realizaron las genotipificaciones por la probabilidad de encontrar más individuos afectados en la familia una vez se aumentara la extensión de las mismas; sin embargo, esto no fue posible dadas las características propias de la enfermedad que complican la aceptación de participación en la investigación. Además, el inicio de la esquizofrenia en la juventud hace difícil que establezcan pareja y que existan, por tanto, grandes familias con múltiples afectados, a diferencia de lo que se observa en otras enfermedades psiquiátricas, como el trastorno bipolar, que causan menos deterioro. Adicionalmente, estas familias viven en áreas rurales de difícil acceso, lo cual aumenta la dificultad para evaluar a todos los individuos e indagar por la existencia de más afectados.

El tamaño de la muestra requerido en los estudios de ligamiento genético y asociación, guarda relación directa con el tamaño del efecto de los genes de susceptibilidad de la enfermedad. Así, cuando menor es el efecto genético, mayor es el tamaño necesario de la muestra. En los estudios de ligamiento, cuando el riesgo relativo es de 4 el número de familias requeridas es de 185 , pero si es de 1,5, como puede ocurrir en esquizofrenia, se necesitarían 4.462.807 familias. ${ }^{30}$ Por tanto, el análisis de ligamiento no es la estrategia más apropiada para detectar genes de efecto pequeño como sucede en la esquizofrenia.

Otro factor que tiene relación con el tamaño de la muestra es la heterogeneidad genética. Cuando está presente, como se espera que suceda en la esquizofrenia, el número necesario de familias para obtener un resultado significativo de ligamiento aumenta. ${ }^{29}$ Sin embargo, en el presente estudio este factor podría no tener un gran efecto teniendo en cuenta que las dos familias son originarias de la misma población $y$, en consecuencia, es posible que en ellas estén presentes los mismos alelos de susceptibilidad si se parte del supuesto de que la población antioqueña es homogénea. ${ }^{31}$

La distancia entre el marcador y el posible gen de susceptibilidad pudo no haber sido un factor importante en el poder de detectar ligamiento ya que en este caso se utilizaron marcadores estrechamente ligados a genes candidatos. Los marcadores del cromosoma 6, D6S1014 y D6S2414 se eligieron por su estrecho ligamiento al gen $\mathrm{NOTCH} 4$. El primero se encuentra en un intrón y el segundo está ubicado a $637 \mathrm{~kb}$ del gen. El marcador del cromosoma 7, D7S2504 se encuentra en un intrón y el segundo está ubicado a $637 \mathrm{~Kb}$ del gen. El marcador del cromosoma 7, D7S2504 se encuentra en un intrón del gen y en cuanto a los marcadores del cromosoma 1, D1S1679 y D1S1653, se sabe que están cerca (menos de $7 \mathrm{Mb}$ ) de genes considerados como candidatos para la enfermedad. Por otro lado, una característica importante de un marcador y propia de los microsatélites es su alto grado de polimorfismo que aumenta la probabilidad de obtener individuos heterocigotos $\mathrm{y}$ encontrar de esta manera meiosis informativas. ${ }^{29}$ Aunque se utilizan marcadores micro- 
satélites para garantizar el poder informativo, éste puede variar de acuerdo con la estructura genética de la población a la cual pertenecen las familias, de tal forma que sólo se puede conocer con exactitud el poder informativo de un marcador cuando se ha genotipificado en dicha población.

Con respecto al poder informativo de los marcadores que se utilizaron, se encontró que en las dos familias, por lo menos uno de los padres de los individuos afectados fue homocigoto para cuatro de los marcadores utilizados, y el único donde se hallaron ambos padres heterocigóticos fue D7S2504 quien mostró el máximo lod score (lod score $=0.18$ ), lo cual redujo el número de meiosis informativas ya que sólo para este último marcador se podrían detectar recombinantes entre un alelo del marcador y el alelo de susceptibilidad en el gen putativo. Adicionalmente, no se incluyeron los genotipos de dos individuos que pudieron haber proporcionado información importante dado que cuando una muestra de estudio es tan pequeña cada individuo aporta información; sin embargo, es poco probable que estos dos individuos hubieran tenido un impacto muy significativo aumentando o disminuyendo demasiado el lod score obtenido.

A todo lo anterior, se suman las complicaciones concernientes a la definición diagnóstica que ha sido uno de los aspectos que más discusión ha generado alrededor del tortuoso proceso de detección de genes de susceptibilidad a las enfermedades complejas. Lo que hoy se llama "Esquizofrenia" cubre un amplio rango de dominios cognitivos, emocionales y del comportamiento; por tanto se está usando una sola palabra para referirse a un trastorno que podría ser clínico, etiológico y fisiopatológicamente heterogéneo. ${ }^{32}$ Por otra parte, si se considerara un patrón de herencia multifactorial con umbral se puede esperar que la combinación de los diferentes genes de susceptibilidad y los factores ambientales origine una gran variedad de manifestaciones. ${ }^{33,34}$ Es posible que en los estudios de ligamiento y asociación no se esté utilizando una adecuada definición del fenotipo, lo cual ha influido enormemente en los resultados de éstos. ${ }^{35,36}$ A este respecto se han propuesto varias estrategias, una de ellas es no analizar el síndrome completo, esquizofrenia, sino subtipos de ella. ${ }^{37}$ Otra aproximación, que tiene gran fuerza actualmente, es el uso de endofenotipos los cuales son las manifestaciones de la susceptibilidad a la enfermedad y comprenden cambios neurofisiológicos, bioquímicos y del comportamiento. Se ha demostrado que al utilizar endofenotipos se aumenta la capacidad de encontrar ligamiento, lo cual posteriormente permitirá la detección de un gen. ${ }^{38}$

También debe tenerse en cuenta que al ser la esquizofrenia una entidad compleja, el análisis de ligamiento no es la aproximación más apropiada para analizar su componente genético por varias razones: 1) Esta estrategia fue diseñada para detectar genes de efecto mayor. 2) Requiere grandes tamaños de muestra. 3) En el caso del análisis paramétrico, que es más poderoso que el no paramétrico, es necesario asumir varios parámetros, como los patrones de herencia y las penetrancias, que pueden o no acercarse a la realidad y, en consecuencia, originar resultados falsos positivos o negativos. Por consiguiente, otras estrategias no paramétricas, como los estudios de asociación, permiten la detección de genes de efecto pequeño utilizando tamaños de muestra más asequibles. ${ }^{30}$

\section{CONCLUSIÓN}

Con el análisis paramétrico y no paramétrico, no fue posible confirmar ni descartar ligamiento entre la esquizofrenia y los mar- 
cadores D1S1653, D1S1679, D6S2414, D6S1014 y D7S2504 estrechamente ligados a genes del neurodesarrollo en estas familias.

Los genes RELN, NOTCH y aquellos localizados en la región 1q21-22, no deben descartarse como posibles genes candidatos en la transmisión de la esquizofrenia, dadas las evidencias importantes halladas en la literatura y las dificultades encontradas en el presente estudio.
Se deben realizar estudios de asociación con muestras de un tamaño apropiado, usar más microsatélites en la región donde se encuentran estos genes y aún mejor usar SNPs (polimorfismo de un único nucleótido) dentro de los genes. Además es importante reducir la heterogeneidad clínica disecando más el fenotipo en endofenotipos, dimensiones de síntomas o subtipos del síndrome. Esta aproximación puede resultar más apropiada en el estudio de enfermedades complejas como la esquizofrenia.

\section{BIBLIOGRAFÍA}

1. American Psychiatric Association. DSMIV-TR: Manual diagnóstico y estadístico de los trastornos mentales. Texto revisado. Washington DC.,2000.

2. World Health Organization. The World Health Report 2001. Mental Health: New understanding, new hope. Geneva, Switzerland: World Health Organization, 2001.

3. McGue M, Gottesman I, Rao D. The Transmision of Schizophrenia Under a Multifactorial Threshold Model. Am. J. Hum. Genet. 1983; 35:1161-78.

4. Miron B. Genetics of Schizophrenia and the New Millennium: Progress and Pitfalls. AM. J. Hum. Genet. 2004; 68:299-312.

5. Hranilovic D, Schwab SG, Jernej B, Knapp M, Lerer B, Albus M. Serotonin transporter gene and schizophrenia: evidence for association/linkage disequilibrium in families with affected siblings. Mol. Psychiatry 2000; 5:91-98.

6. O'Donovan M, Owen J. Candidate Gene Association Studies of Schizophrenia. Am. J. Hum. Genet. 1999; 65:587-92.
7. Tsuang M. Schizophrenia: genes and environment. Biol. Psychiatry 2000; 47:210-20.

8. Tsuang MT, Stone WS, Faraone SV. Genes, environment and schizophrenia. Br. J. Psychiatry Suppl. 2001; 40s: 18-24.

9. Lewis DA, Levitt P. Schizophrenia as a disorder of neurodevelopment. Annu. Rev. Neurosci. 2002; 25:409-32.

10. Eastwood SL, Harrison PJ. Interstitial white matter neurons express less reelin and are abnormally distributed in schizophrenia: towards an integration of molecular and morphologic aspects of the neurodevelopmental hypothesis. Mol. Psychiatry 2003; 8:769, 821-769, 831.

11. Akahane A, Kunugi H, Tanaka H, Nanko S. Association analysis of polymorphic CGG repeat in 5' UTR of the reelin and VLDLR genes with schizophrenia. Schizophr. Res. 2002; 58:37-41.

12. Wassink T, Nopoulos P, Pietila J. NOT$\mathrm{CH} 4$ and the frontal lobe in schizophrenia. AM. J. Med. Genet. 2003; 118B:1-/. 
13. Barinaga M. Guidin neurons to the cortex. Science 1996; 274:1100-01.

14. Magdaleno S, Keshvara L, Curran T. Rescue of ataxia and preplate splitting by ectopic expression of reelin in releer mice. Neuron 2002; 33:573-86.

15. Dulabon L, Olson EC, Taglienti MG, Eisenhuth S, McGrath B, Walsh CA. Reelin binds alpha-3-beta-1 integrin and inhibits neuronal migration. Neuron 2000; 27:33-44.

16. Fatemi SH, Kroll JL, Stary JM. Altered levels of Reelin and its isoforms in schizophrenia and mood disorders. Neuroreport 2001; 12:3209-15.

17. Chen ML, Chen SY, Huang $\mathrm{CH}$, Chen $\mathrm{CH}$. Identification of a single nucleotide polymorphism at the $5^{\prime}$ promoter region of human reelin gene and association study with schizophrenia. Mol. Psychiatry 2002; 7:447-48.

18. Impagnatiello F, Guidotti AR, Pesold C, Dwivedi Y, Caruncho H, Pisu MG et al. A decrease of reelin expression as a putative vulnerability factor in schizophrenia. Proc Natl Acad Sci USA 1998; 95:157/18-23.

19. Artavanis-Tskonas S. Notch signiling: cell fate control and signal integration in development. Science 1999; 284:770-76.

20. Wei J, Hemmings GP. The NOTCH4 locus is associated with susceptibility to schizophrenia. Nat. Genet. 2000; 25:376-77.

21. Jurewicz I, Owen RJ, O'Donovan MC, Owen MJ. Searching for susceptibility genes in schizophrenia. Eur. Neuropsychopharmacol. 2004; 11:395-98.

22. Brzustowicz LM, Hodgkinson KA, Chow EW, Honer WG, Bassett AS. Location of a major susceptibility locus for familial schizophrenia on chromosome 1q21-q22. Science 2000; 288:678-82.

23. Fañañas R, Peralta M, Sham P. 1q21-22 locus is associated with susceptibility to the reality distortion syndrome of schizophrenia spectrum disorders. Am. J. Med. Genet. 2002; 114:516-18.

24. Mirnics K, Middleton FA, Lewis DA, Levitt P. Analysis of complex brain disorders with gene expression microarrays: schizophrenia as a disease of the synapse. Trends Neurosci. 2001; 24:47986.

25. Mirnics K, Lewis DA. Genes and subtypes of schizophrenia. Trends Mol. Med. 2001; 7:281-83.

26. Miurna Y, Mardy S, Awaya Y, Nihei K, Endo F, Matsuda I. Mutation and Polymorphism Analysis of the TRKA (NTRK1) Gene Encoding a High-Affinity Receptor for Nerve Growth Factor in Congenital Insensitivity to Pain With Anhidrosis (CIPA) Families. Hum. Genet. 2001;106: 116-24.

27. Nurnberger JI, Blehar MC, Kaufmann C, York-Cooler C, Simpson SG, HarkavyFriedman J et al. Diagnostic Interview for Genetic Studies: rationales, unique features and training. Arch. Gen. Psychiatry 1994; 51:849-59.

28. Palacio CA, García J, Arbeláez MP, Sánchez R, Aguirre B, Garcés I et al. Validación de la entrevista diagnóstica para estudios genéticos (DIGS) en Colombia. Biomédica 2004; 24:56-62.

29. Haines JH, Pericak-Vance MA. Approaches to gene mapping in complex human diseases. New York: Wiley-Liss Inc; 1998 p. 1-434. 
30. Risch N, Merikangas K. The Future of Genetic Studies of Complex Human Diseases. Science 1996; 273:1516-17.

31. Soto, I. D. Evaluación histórica y genética del origen de Marinilla y su zona de influencia. 2003. Universidad de Antioquía.

\section{Tipo Ref. Tesis}

32. Andreasen N, Roy M, Flaum M. Positive and negative symptoms., In: Steven R Hirsch and Daniel R Weinbeerger, editor. Schizophrenia. Oxford.: Blackwell Science; 1995. p. 28-45.

33. Moller H. Bipolar Disorder and Schizophrenia: Distinct Illnesses or a Continuum. J. Clin. Psychiatry 2003; 64:23-27.

34. Tsuang MT, Stone WS, Faraone SV. Toward reformulating the diagnosis of schizophrenia. Am. J. Psuchiatry 2000; $157: 1041-50$.

35. Gottesman II, Gould T. The endophenotype concept in psychiatry: etymology and strategic intention. Am. J. Psychiatry 2003; 160:363-45.

36. Maier W. Psychiatric genetics. Overview on achievements, problems, perspective. Methods Mol. Med. 2003;77:20.

37. Stober G, Saar K, Ruschendorf F, Meyer J, Nurnberg G, Jatzke S. Splitting schizophrenia: periodic catatonia-susceptibility locus on chromosome 15q 15. Am. J. Hum. Genet. 2000;67:1201-07.

38. Freedman R, Adler LE, Leonard S. Alternative phenotypes for the complex genetics of schizophrenia. Biol. Psychiatry 1999; 45:551-58.

* Autor responsable de la correspondencia: Jorge Ospina-Duque. Coordinador del Programa de Investigación en Psiquiatría Genética y Jefe del Departamento de Psiquiatría. Facultad de Medicina, Universidad de Antioquia. Dirección postal: Calle 2 sur \# 46-55, Consultorio 335. Clínica Las Vegas. Medellín, Colombia. Tel.: (57-4) 311.8039. Fax: (57-4) 312.7512. Dirección electrónica: jhospina@epm.net.co 